\title{
Pharmacokinetics of Nimustine, Cytosine Arabinoside, and Methotrexate in Cerebrospinal Fluid during Cerebrospinal Fluid Perfusion Chemotherapy
}

\author{
Norifumi Morikawa, ${ }^{* a}$ Teruaki Mori,${ }^{b}$ Hisanori Kawashima, ${ }^{a}$ Masaharu Takeyama, ${ }^{a}$ Tatsuya Abe, ${ }^{b}$ \\ and Hidenori KOBAYASHI ${ }^{b}$
}

Departments of Clinical Pharmacy ${ }^{a}$ and Neurosurgery, ${ }^{b}$ Oita Medical University, Hasama-machi, Oita 879-5593, Japan.

Received Septembre 4, 2000; accepted December 5, 2000

This report investigates the pharmacokinetics of nimustine (ACNU), cytosine arabinoside (Ara-C), and methotrexate (MTX) in cerebrospinal fluid (CSF) during CSF perfusion chemotherapy. A 47-year-old Japanese man with spinal cord, cerebellum and brain stem dissemination of oligo-astrocytoma received nine courses of CSF perfusion chemotherapy with ACNU, Ara-C, and MTX. A CSF perfusion chemotherapy solution was perfused via an Ommaya reservoir in the ventricle, and was discharged by drainage though another Ommaya reservoir in the lumbar spinal canal. CSF samples via Ommaya reservoirs in the lumbar spinal canal were obtained during the fifth and eighth courses of treatment. The concentrations of ACNU and Ara-C in CSF were measured by HPLC, and the MTX concentrations by fluorescence polarization immunoassay. In the fifth course of treatment, a CSF injection chemotherapy solution, consisting of $5 \mathrm{mg}$ of ACNU dissolved in $20 \mathrm{ml}$ of artificial CSF, was injected over a few minutes using the Ommaya reservoir. Next, a CSF perfusion chemotherapy solution, consisting of $10 \mathrm{mg}$ of Ara-C and $5 \mathrm{mg}$ of MTX dissolved in $100 \mathrm{ml}$ of artificial CSF, was perfused over $2 \mathrm{~h}$. In the eighth course of treatment, a CSF perfusion chemotherapy solution, consisting of $5 \mathrm{mg}$ of ACNU, $10 \mathrm{mg}$ of Ara-C and $5 \mathrm{mg}$ of MTX dissolved in $100 \mathrm{ml}$ of artificial CSF, was perfused over $2 \mathrm{~h}$. In both treatments, the highest concentrations of Ara-C and MTX in CSF were observed 1 or $2 \mathrm{~h}$ after the end of perfusion, with the values of each drug being similar. The CSF AUCs of Ara-C and MTX in each treatment were of similar values. Although the highest concentration of ACNU in CSF was observed in the fifth treatment $1 \mathrm{~h}$ after injection (an injection chemotherapy of ACNU plus a perfusion chemotherapy of Ara-C and MTX), the concentration of ACNU in CSF was undetectable in the eighth treatment (a perfusion chemotherapy of ACNU, Ara-C and MTX). We were successful in administering all anticancer drugs, and reaching a level of over $1.0 \mu \mathrm{g} / \mathrm{ml}$ concentration in CSF of the lumbar spinal canal, using an injection chemotherapy of ACNU plus a perfusion chemotherapy of Ara-C and MTX; this was done even though the drugs, in particular ACNU, underwent some perfusion-period dependent decomposition.

Key words cerebrospinal fluid perfusion chemotherapy; pharmacokinetics; nimustine; cytosine arabinoside; methotrexate

The treatment of disseminated malignant brain tumors generally involves intrathecal chemotherapy with methotrexate (MTX) alone, or in combination with cytosine arabinoside (Ara-C), together with local radiotherapy. ${ }^{1-5)}$ However, because maximum doses of MTX are limited by neurotoxicity, clinical results have not been satisfactory. ${ }^{6)}$ Recently a pilot study evaluating the efficacy and side effects of cerebrospinal fluid (CSF) perfusion chemotherapy with Ara-C and MTX in patients with meningeal carcinomatosis found that this therapy was effective in improving cerebral, cranial nerve and spinal root signs and symptoms. ${ }^{7)}$ In addition, we have reported on the pharmacokinetics of nimustine (ACNU), Ara-C, and MTX in CSF during CSF perfusion chemotherapy in three patients with disseminated brain tumors. ${ }^{8,9)}$ We found that CSF perfusion chemotherapy enables high concentrations of anticancer drugs to be administered for dissemination in the spinal cord within a short period of time, and with minimal adverse effects. In addition, the maximum concentrations of anticancer drugs in discharged CSF and the duration of perfusion were inversely correlated. Further, concentrations of ACNU in the lumbar area were not detectable during CSF perfusion chemotherapy of more than $2 \mathrm{~h}$ duration. However, as our aim was to retain all administered anticancer drugs within the spinal cord covered by the disseminated brain tumor, we proposed a new protocol for CSF perfusion chemotherapy: a CSF injection chemotherapy of ACNU plus a CSF perfusion chemotherapy of Ara-C and MTX. In the present study, we investigated the pharmacokinetics in CSF during the new protocol of CSF perfusion chemotherapy in a patient with a disseminated brain tumor.

\section{MATERIALS AND METHODS}

Chemicals ACNU and Ara-C were kindly supplied by Sankyo (Tokyo, Japan) and Nippon Shinyaku (Kyoto, Japan), respectively. Methanol and acetonitrile were of HPLC grade. All other chemicals were of analytical grade. ACNU (Nidran $^{\mathrm{TM}}$ injection), Ara-C (Cylocide ${ }^{\mathrm{TM}}$ injection), and MTX (Methotrexate ${ }^{\mathrm{TM}}$ parenteral) were purchased from Sankyo Co., Ltd. (Tokyo), Nihon Shinyaku Co., Ltd. (Kyoto), and Wyeth Lederle Japan, Ltd. (Tokyo), respectively.

Patient A 37-year-old Japanese man (162 cm, $54 \mathrm{~kg}$ ) was diagnosed with mixed oligo-astrocytoma and underwent subtotal removal of a left frontal tumor by osteoplastic craniotomy in April 1986. He then received radiation therapy, consisting of 30 gray (Gy) to the whole brain and 30 Gy to the frontal brain, in May and June. After this therapy he was discharged in July 1986. In June 1996 at age 47 he felt a headache and was dizzy. In August of that year, metastatic diseases in the right frontal pole, stalk, supracellebellar cistern, vermis and fourth ventricle were suspected following magnetic resonance imaging (MRI) examination, and he was 
admitted for further treatment. In September, Ommaya reservoirs were placed in the ventricle and lumbar spinal canal. By the end of September, a total of nine courses of CSF perfusion chemotherapy using ACNU, Ara-C, and MTX had been administered. Informed written consent for the treatment had been obtained from the patient. A CSF perfusion chemotherapy solution was perfused via an Ommaya reservoir in the ventricle and was discharged by drainage through another Ommaya reservoir in the lumbar spinal canal. The concentrations of the anticancer drugs in CSF of this canal were measured during the fifth and eighth courses of treatment. In the fifth course of treatment, a CSF injection chemotherapy solution, consisting of $5 \mathrm{mg}$ of ACNU dissolved in $20 \mathrm{ml}$ of artificial CSF ( $19.9 \mathrm{ml}$ of lactated Ringer's, $0.08 \mathrm{ml}$ of $7 \%$ sodium bicarbonate, and $0.02 \mathrm{ml}$ of $50 \%$ glucose) was injected using the Ommaya reservoir a few minutes after dissolution in artificial CSF. Next, a CSF perfusion chemotherapy solution, consisting of $10 \mathrm{mg}$ of Ara-C and $5 \mathrm{mg}$ of MTX dissolved in $100 \mathrm{ml}$ of artificial CSF, was perfused over $2 \mathrm{~h}$. In the eighth course of treatment, a CSF perfusion chemotherapy solution, consisting of $5 \mathrm{mg}$ of ACNU, $10 \mathrm{mg}$ of Ara-C, and $5 \mathrm{mg}$ of MTX dissolved in $100 \mathrm{ml}$ of artificial CSF, was perfused over $2 \mathrm{~h}$ immediately after its dissolution in artificial CSF. CSF samples were obtained, using the Ommaya reservoir, by lumbar drainage at baseline and at $0.5,1.0,1.5,2.0,3.0,4.0,6.0$, and $24 \mathrm{~h}$ after the initiation of perfusion. The CSF samples $(2.0 \mathrm{ml})$ were collected in tubes, frozen immediately and stored at $-20{ }^{\circ} \mathrm{C}$ until analysis. The concentrations of ACNU and Ara-C in CSF were measured by HPLC assay according to methods previously reported. ${ }^{8)}$ The concentrations of MTX in CSF were measured by a fluorescence polarization immunoassay (FPIA, Tdx Abbott, North Chicago, IL, U.S.A.) according to a previously reported method. ${ }^{8)}$ Values for the area under the time-concentration curve from 0 to $24 \mathrm{~h}\left(A U C_{0-24}\right)$ for anticancer drugs were calculated by the trapezoidal rule. The patient showed a partial clinical response according to MRI performed 3 months after the first treatment, and there were no complications related to treatment. However, he abruptly developed respiratory and cardiac arrest in December. After being revived, he had conservative treatment but died in February 1997.

\section{RESULTS}

The CSF concentrations of anticancer drugs on the fifth and eighth courses of treatments are shown in Figs. 1 and 2. No anticancer drugs were detectable in CSF at the initiation of each perfusion.

In the fifth course of treatment, during CSF injection chemotherapy of ACNU plus CSF perfusion chemotherapy using Ara-C and MTX, the highest concentrations of Ara-C $(5.55 \mu \mathrm{g} / \mathrm{ml})$ and MTX $(6.28 \mu \mathrm{g} / \mathrm{ml})$ in CSF were observed at $1 \mathrm{~h}$ after the end of the perfusion. The highest concentration of ACNU in CSF was also observed at $1 \mathrm{~h}$ after injection $(1.08 \mu \mathrm{g} / \mathrm{ml})$, and then decreased to $0.26 \mu \mathrm{g} / \mathrm{ml}$ at the end of the perfusion. The CSF $A U C_{0-24}$ of $\mathrm{ACNU}$, Ara-C, and MTX, was $1.08,45.64$, and $111.43 \mu \mathrm{g} \cdot \mathrm{h} / \mathrm{ml}$, respectively (Fig. 1).

In the eighth course of treatment, during CSF perfusion chemotherapy using ACNU, Ara-C and MTX, the highest

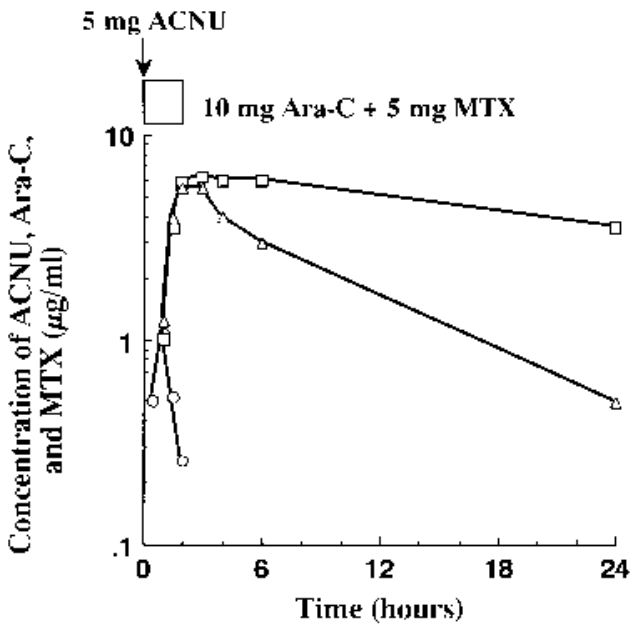

Fig. 1. Time Courses of Concentrations of Anticancer Drugs in CSF Following the Fifth Treatment, a CSF Injection Chemotherapy of $5 \mathrm{mg}$ of ACNU Plus a CSF Perfusion Chemotherapy Using $10 \mathrm{mg}$ of Ara-C and $5 \mathrm{mg}$ of MTX

ACNU, $\bigcirc$; Ara-C, $\triangle$; MTX, $\square$. Time: time after the start of perfusion.

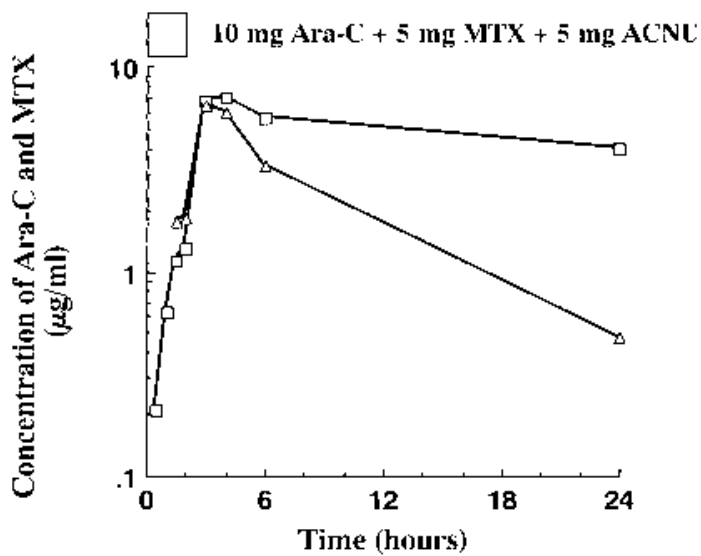

Fig. 2. Time Courses of Concentrations of Anticancer Drugs in CSF Following the Eighth Treatment, a CSF Perfusion Chemotherapy Using $5 \mathrm{mg}$ of ACNU, $10 \mathrm{mg}$ of Ara-C and $5 \mathrm{mg}$ of MTX

Ara-C, $\triangle$; MTX, $\square$. Time: time after the start of perfusion.

concentrations of Ara-C $(6.48 \mu \mathrm{g} / \mathrm{ml})$ and MTX $(7.08 \mu \mathrm{g} / \mathrm{ml})$ in CSF were observed 1 or $2 \mathrm{~h}$ after the end of the perfusion, but no concentration of ACNU in CSF was observed. The CSF $A U C_{0-24}$ of Ara-C and MTX was 46.08 and $110.04 \mu \mathrm{g}$. $\mathrm{h} / \mathrm{ml}$, respectively (Fig. 2).

\section{DISCUSSION}

In the present study, we investigated the pharmacokinetics of ACNU, Ara-C, and MTX in lumbar CSF during and after CSF perfusion chemotherapy in a patient with disseminated glioma. Meningeal carcinomatosis is generally treated by intrathecal chemotherapy with MTX alone or in combination with Ara-C. ${ }^{1-5)}$ However, Bleyer ${ }^{10)}$ and Shapiro et al. ${ }^{11)}$ have reported that when MTX is administered by lumbar puncture, the concentration of MTX that arrives at the subarachnoid space is low. In addition, Rieselbach et al. ${ }^{12)}$ proposed that lumbar injections of Ara-C and MTX do not sufficiently distribute these drugs in the subarachnoid space of the brain. Similar assumptions have been made about chemotherapy 
drugs injected through an Ommaya reservoir. We have also reported that concentrations of ACNU were not detectable in the lumbar spinal canal on injection into ventricle CSF of $10 \mathrm{mg}$ of ACNU using an Ommaya reservoir. ${ }^{13)}$ We thus realized the necessity of developing a new treatment method for patients with disseminated brain tumors in the spinal canal.

Nakagawa et al. reported that 9 of 13 patients with meningeal carcinomatosis, who were treated by CSF perfusion chemotherapy using Ara-C and MTX, showed positive responses. ${ }^{7)}$ We also demonstrated that high concentrations of Ara-C and MTX arrived at the lumbar spinal canal during CSF perfusion chemotherapy using Ara-C and MTX within a short period of time, thus allowing for effective drug concentrations to be maintained in the CSF for an extended period. ${ }^{8)}$ By contrast, when ACNU was administered by CSF perfusion chemotherapy, it was initially detectable in the CSF of the injection site at the end of perfusion but was not detectable $8 \mathrm{~h}$ after the end of perfusion, most likely because the half-life of ACNU is very short. We also reported that the final half-lives of ACNU, Ara-C, and MTX during CSF perfusion chemotherapy are about 1,2 , and $4 \mathrm{~h}$, respectively, because the half-lives were gradually decreased with the decrease in protein values in CSF after each treatment. Furthermore, even at the injection sites, the CSF concentrations of ACNU decreased to less than $1 \mu \mathrm{g} / \mathrm{ml}$ at $3 \mathrm{~h}$ after the end of perfusion. ${ }^{8)}$ In an experiment involving the intraventricular injection of ACNU in dogs, Levin et al. reported that a significant amount of ACNU in the CSF is hydrolytically degraded before reaching subarachnoid regions distal to the injection site. ${ }^{14)}$ We previously reported similar results with respect to the decomposition of ACNU in humans, and that the maximum concentration of ACNU in lumbar discharged CSF had degraded to less than $1 \%$ of the concentration of the original perfusion solution after $3 \mathrm{~h}$ of CSF perfusion chemotherapy due to the extremely short half-life of ACNU, taking into account the rapid degradation of ACNU in solution at body temperature. ${ }^{8)}$ These results indicated that CSF perfusion chemotherapy with anticancer drugs, including ACNU, should be performed within $1 \mathrm{~h}$, and that ACNU should be immediately perfused after dissolution in artificial CSF. However, many patients require more than a $2 \mathrm{~h}$ perfusion period for CSF perfusion chemotherapy. In order to deliver ACNU to the lumbar spinal canal, we thus believed it would be necessary to develop a new protocol.

We tested a new protocol of CSF injection chemotherapy of ACNU, plus CSF perfusion chemotherapy using Ara-C and MTX in a patient with disseminated glioma, and mea- sured the concentrations of the anticancer drugs in CSF. In the present study, a $2 \mathrm{~h}$ perfusion period was used for all treatments. We have reported that the transition of ACNU into CSF was noted after $5 \mathrm{~min}$, showing a maximum value of $0.59 \pm 0.13 \mu \mathrm{g} / \mathrm{ml}$ after $30 \mathrm{~min}$, when $100-150 \mathrm{mg} /$ body of ACNU was injected intravenously in 14 cases of brain tumors. ${ }^{15)}$ We aimed to reach over $1.0 \mu \mathrm{g} / \mathrm{ml}$ of ACNU concentration within the spinal cord covered with the disseminated brain tumor. We were successful in delivering all the administered anticancer drugs, reaching over $1.0 \mu \mathrm{g} / \mathrm{ml}$ concentration in CSF of the lumbar spinal canal, using an injection chemotherapy of ACNU plus a perfusion chemotherapy of Ara-C and MTX. However, the drugs, in particular ACNU, did undergo some perfusion-period dependent decomposition. This protocol may be useful for many patients with disseminated brain tumors. In the present study, we could not identify the distribution profiles of the concentrations of the anticancer drugs in spinal canal CSF. Future studies of appropriate concentrations of the perfused anticancer drugs, and the possible synergistic effects of drug combinations, are necessary.

\section{REFERENCES}

1) Yap H., Yap B., Rasmussen S., Levens M. E., Hortobagyi G. N., Blumenschein G. R., Cancer, 49, 219-222 (1982).

2) Wasserstom W. R., Glass J. P., Posner J. B., Cancer, 49, 759-772 (1982).

3) Giannone L., Greco F. A., Hainsworth J. D., J. Clin. Oncol., 4, 68-73 (1986).

4) Hitchines R. N., Bell D. R., Woods R. L., Levi J. A., J. Clin. Oncol., 5, 1655-1662 (1987).

5) Theodore W. H., Gendelman S., Arch. Neurol., 38, 696-699 (1981).

6) Bleyer W. A., Drake J. C., Chabner B. A., N. Engl. J. Med., 289, 770773 (1973).

7) Nakagawa H., Fujita T., Kubo S., Izumoto S., Nakajima Y., Tsuruzono K., Tokiyoshi K., Hayakawa T., Surg. Neurol., 45, 256-264 (1996).

8) Morikawa N., Mori T., Kawashima H., Fujiki M., Abe T., Kaku T., Konisi Y., Takeyama M., Hori S., Eur. J. Clin. Pharmacol., 54, 415420 (1998).

9) Morikawa N., Mori T., Kawashima H., Takeyama M., Hori S., Ann. Pharmacother., 32, 1008-1012 (1998).

10) Bleyer W. A., Natl. Cancer. Inst. Monogr., 46, 171-178 (1977).

11) Shapiro W. R., Young D. F., Mehta B. M., N. Engl. J. Med., 293, 161166 (1975).

12) Rieselbach R. E., Chiro G. D., Freireich E. J., Rall D. P., N. Engl. J. Med., 267, 1273-1278 (1962).

13) Morikawa N., Mori T., Fujiki M., Abe T., Kawashima H., Takeyama M., Hori S., Jpn. J. Hosp. Pharm., 25, 376-384 (1999).

14) Levin V. A., Byrd D., Campbell J., Giannini D. D., Borcich J. K., Davis R. L., Cancer Res., 45, 3803-3809 (1985).

15) Mori T., Mineura K., Katakura R., Noshinkei, 31, 601-606 (1979). 